\title{
Analytical Study of the Ekman Angle for the Isothermal Flow of a Viscous Incompressible Fluid in View of the Navier Boundary Condition
}

\author{
A. V. Gorshkov ${ }^{1,2, a)}$ and E. Yu. Prosviryakov ${ }^{1,2, b)}$ \\ ${ }^{1}$ Institute of Engineering Science, Ural Branch of the Russian Academy of Sciences, 34 Komsomolskaya St., \\ Ekaterinburg, 620049, Russia \\ ${ }^{2}$ B. N. Yeltsin Ural Federal University, 19 Mira St., Ekaterinburg, 620002, Russia

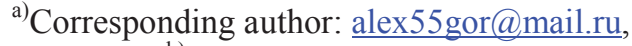 \\ b)evgen_pros@mail.ru
}

\begin{abstract}
The Ekman angle is the angle between the tangential stress vector on the free surface and the flow velocity vector in the upper layer. Ekman showed that, for an isobaric flow in an ocean with an infinitely great depth (fluid flow in half-space), the angle is equal to $45^{\circ}$. The paper studies the Ekman angle occurring in an isothermal laminar flow with the Navier slip boundary condition. In order to determine the Ekman angle, an analytical solution of the Oberbeck-Boussinesq equations is constructed; the solution describes the laminar Ekman-Poiseuille isothermal flow with allowance made for two components of the Coriolis force and the Navier condition at the lower (solid) boundary. The components of the tangential stress vector are set at the upper boundary. The velocity representation in the form of linear functions of the horizontal coordinates is used. The paper studies the dependence of the Ekman angle on the pressure gradient and on the friction coefficient on the solid surface, the depth of the ocean being finite. It is shown that at a sufficiently large depth there is no friction effect, and the Ekman angle is $45^{\circ}$.
\end{abstract}

\section{INTRODUCTION}

The study of rotating fluids is a central problem of hydrodynamics [1]. The study of large-scale currents of the world's ocean and air motions in the Earth's atmosphere formed a relatively young science of geophysical hydrodynamics [2].

The analytical study of the angle between the vector of tangential stresses at the flow boundary, which simulate the action of wind, and the fluid flow velocity vector (hereinafter referred to as the Ekman angle) is an important task of geophysical hydrodynamics [1].

When calculating the world's ocean currents, one uses the conventional approximation of the Coriolis acceleration, which is characterized by a single parameter. Strictly speaking, this approach allows one to describe fluid motion at high latitudes [2-5]. As is known, this approach is also used at middle latitudes since in large currents the horizontal velocities exceed the vertical one by several orders of magnitude [5]. The generalization of the Ekman shear flow and the study of its stability were discussed in [2, 3, 5-16].

The studies of the Ekman angle are based on exact solutions to nonlinear Navier-Stokes equations describing laminar fluid flows with an inclined axis of rotation. In most studies, it is assumed that the condition of no-slip of a viscous fluid to a solid surface is met. It is assumed that the fluid moves relative to a solid surface and that the interaction force is described by Navier's law. 


\section{PROBLEM STATEMENT}

A large-scale steady-state laminated isothermal flow of a rotating viscous incompressible fluid is described by a system of Navier-Stokes equations taking into account the Coriolis force. The system of equations written in dimensionless variables in view of the fact that $V_{z}=0$ has the following form $[2,15,16]$ :

$$
\begin{gathered}
\operatorname{Re}\left(V_{x} \frac{\partial V_{x}}{\partial x}+V_{y} \frac{\partial V_{x}}{\partial y}\right)-\frac{\sin \varphi}{E k} V_{y}=-\frac{\operatorname{Re}}{\delta} \frac{\partial P}{\partial x}+\Delta V_{x}, \\
\operatorname{Re}\left(V_{x} \frac{\partial V_{y}}{\partial x}+V_{y} \frac{\partial V_{y}}{\partial y}\right)+\frac{\sin \varphi}{E k} V_{x}=-\frac{\operatorname{Re}}{\delta} \frac{\partial P}{\partial y}+\Delta V_{y}, \\
\operatorname{Re} \frac{\partial P}{\partial z}=\frac{\cos \varphi}{E k} V_{x}, \frac{\partial V_{x}}{\partial x}+\frac{\partial V_{y}}{\partial y}=0 .
\end{gathered}
$$

Here, $V_{x}, V_{y}$ are the dimensionless components of the fluid velocity vector; $P$ is the pressure normalized to twice the specific kinetic energy $\rho V^{2}$; the dimensionless horizontal coordinates $x$ and $y$ are determined by the characteristic length scale $L$; the transverse coordinate $z$ is determined by the fluid layer thickness $h ; \delta=h / L$ is the ratio of the length scales; $\operatorname{Re}=W L / v$ is the Reynolds number; $\operatorname{Re}=W L / v$ is the Ekman number; $W$ is the characteristic velocity of the fluid flow; $\Omega$ is the angular velocity of fluid rotation; $v$ is the kinematic viscosity of the fluid; $\varphi$ is the latitude of the place; $\Delta^{*}=\frac{\partial^{2}}{\partial x^{2}}+\frac{\partial^{2}}{\partial y^{2}}+\frac{1}{\delta^{2}} \frac{\partial^{2}}{\partial z^{2}}$ is the three-dimensional Laplace operator.

At the lower (solid) boundary, the Navier friction condition is specified; at the upper (free) boundary, tangential stresses simulating the wind action are set.

The analysis of system (2) shows that the solvability of the system is possible in the simplest polynomial class of Lin-Sidorov-Aristov solutions [6-14],

$$
\begin{gathered}
V_{x}=U(z), V_{y}=V(z), \\
P=P_{0}(z)+x P_{1}(z)+y P_{2}(z) .
\end{gathered}
$$

Having substituted solution (2) into equation system (1), we obtain the following resolving system of ordinary differential equations for the functions $U, V, P_{0}, P_{1}$, and $P_{2}$ :

$$
\begin{gathered}
P_{1}^{\prime}=0, P_{2}^{\prime}=0 \\
U^{\prime \prime}=\delta^{2} \operatorname{Re} P_{1}-\frac{\delta^{2} \sin \varphi}{\mathrm{Ek}} V, V^{\prime \prime}=\delta^{2} \operatorname{Re} P_{2}+\frac{\delta^{2} \sin \varphi}{\mathrm{Ek}} U \\
P_{0}^{\prime}=\frac{\delta \cos \varphi}{\operatorname{Re} E \mathrm{k}} U .
\end{gathered}
$$

Here, the prime denotes differentiation with respect to $z$.

The equation system (3)-(5) can be successively integrated in the order the equations are written. It follows from Eq. (3) that the horizontal pressure gradients $P_{1}$ and $P_{2}$ are constant across the layer thickness.

When this class of exact solution (2) for system (1) is used, the second Coriolis parameter is involved only in the equation for determining the pressure $P_{0}$, i.e. Eq. (5); the velocity field defined by Eq. (4) is a generalization of the classical Ekman flow [6-19]. It follows from Eq. (5) that, when two Coriolis parameters are taken into account, the laminar flow of the fluid is not isobaric in the rotating coordinate system. In other words, if the second Coriolis parameter is not ignored in the calculation of flows, the hydrostatic approximation of the equations of oceanology is certainly not fulfilled.

The equations of system (4) with $\sin \varphi=0$ have solutions of another type. Therefore, the case of the current near the equator $(\varphi=0)$ should be considered separately. 
To find a particular solution to system (5)-(6), we set the following boundary conditions: the Navier friction conditions are set at the lower boundary defined by the equation $z=0[20,21]$,

$$
a U^{\prime}=\delta U, a V^{\prime}=\delta V,
$$

where $a=\alpha / L$ is the dimensionless slip length; $\alpha$ is the Navier friction coefficient.

Tangential stresses and pressure are taken into account at the upper boundary defined by the equation $z=1[1,16]$,

$$
U^{\prime}=\delta \tau_{1}, V^{\prime}=\delta \tau_{2}, P_{0}=S
$$

Here, $\tau_{1}$ and $\tau_{2}$ are the projections of the tangent stress vector onto the axes $x$ and $y$, respectively. With the axis $o x$, the tangential stress vector forms the angle $\vartheta_{\tau}=\arctan \left(\tau_{2} / \tau_{1}\right)$. The pressure is assumed to be uniform, independent of the horizontal coordinates.

The selection of the boundary condition (6) allows different types of boundary conditions set at the bottom of a deep water reservoir to be studied from a single point of view $(z=0)$. As a rule, oceanology currently deals with the no-slip condition $(a=0)[2,3,5]$ or the perfect slip condition $(a=\infty)$. In view of the fact that that there is a thin layer of granulated surface on the ocean floor, the relationships from Eq. (6) are used to take it into account.

\section{THE POISEUILLE-COUETTE-EKMAN FLOW CHARACTERIZED BY TWO CORIOLIS PARAMETERS}

The system of differential equations (4) is linear and inhomogeneous. The eigenvalue equation of the corresponding linear homogeneous system is the fourth-order algebraic equation

$$
\lambda^{4}+4\left(\frac{\sin \varphi}{2 \mathrm{Ek}}\right)^{2}=0
$$

The eigenvalues of the system of equations (4), which are the roots of equation (8), are equal to

$$
\lambda_{1}=k(1+i), \lambda_{2}=k(1-i), \lambda_{3}=k(-1+i), \lambda_{4}=k(-1-i),
$$

where, for brevity, it is denoted that $k=\sqrt{\frac{|\sin \varphi|}{2 E k}}$. The modulus $|\sin \varphi|$ is taken with the consideration that $\varphi<0$ in the Southern Hemisphere and that equation (8) involves $\sin \varphi$ squared. The general solution of system (5) has the following form:

$$
\begin{gathered}
U=\left(c_{1} e^{k z \delta}+c_{3} e^{-k z \delta}\right) \cos (k z \delta)+\left(c_{2} e^{k z \delta}+c_{4} e^{-k z \delta}\right) \sin (k z \delta)-\frac{\operatorname{ReEk} P_{2}}{\sin \varphi}, \\
V=\left(c_{4} e^{-k z \delta}-c_{2} e^{k z \delta}\right) \cos (k z \delta)+\left(c_{1} e^{k z \delta}-c_{3} e^{-k z \delta}\right) \sin (k z \delta)+\frac{\operatorname{ReEk} P_{1}}{\sin \varphi}
\end{gathered}
$$

where $c_{1}, c_{2}, c_{3}$, and $c_{4}$ are arbitrary integration constants found from the boundary conditions.

The solution of equation (5) is found by integrating its right-hand side,

$$
P_{0}=2 P_{2} z \delta \operatorname{ctg} \varphi+\frac{\cos \varphi}{\operatorname{EkRe} k}\left[\left(\left(c_{3}-c_{4}\right) e^{-k \delta z}+\left(c_{1}+c_{2}\right) e^{k \delta z}\right) \sin (k \delta z)-\left(\left(c_{3}+c_{4}\right) e^{-k \delta z}+\left(c_{2}-c_{1}\right) e^{k \delta z}\right) \cos (k \delta z)\right]+S .
$$


A particular solution of system (4) satisfying the given boundary conditions (6), (7) will take the following form:

$$
\begin{gathered}
U(z)=\frac{\tau_{1}-\tau_{2}}{2 k}\left[f_{6}(z)+f_{7}(z)+2 a k\left(f_{2}(z)+f_{3}(z)\right)+2 a^{2} k^{2}\left(f_{6}(z)-f_{7}(z)\right)\right]+ \\
+\frac{\tau_{1}+\tau_{2}}{2 k}\left[f_{5}(z)+f_{8}(z)+2 a k\left(f_{1}(z)-f_{4}(z)\right)+2 a^{2} k^{2}\left(f_{5}(z)-f_{8}(z)\right)\right]+ \\
+\frac{P_{1} \mathrm{Gr}}{2 k^{2}}\left[a k\left(f_{6}(z-1)-f_{5}(z-1)+f_{8}(z-1)-f_{7}(z-1)\right)+f_{3}(z-1)+f_{4}(z-1)\right]+ \\
+\frac{P_{2} G r}{2 k^{2}}\left[-1+f_{1}(z-1)+f_{2}(z-1)+a k\left(f_{6}(z-1)+f_{5}(z-1)-f_{8}(z-1)-f_{7}(z-1)\right)\right], \\
V(z)=\frac{\tau_{1}+\tau_{2}}{2 k}\left[2 a k\left(f_{2}(z)+f_{3}(z)\right)+f_{6}(z)+f_{7}(z)+2 a^{2} k^{2}\left(f_{6}(z)-f_{7}(z)\right)\right]- \\
-\frac{\tau_{1}-\tau_{2}}{2 k}\left[2 a k\left(f_{1}(z)+f_{4}(z)\right)+f_{5}(z)+f_{8}(z)+2 a^{2} k^{2}\left(f_{5}(z)-f_{8}(z)\right)\right]+ \\
+\frac{P_{2} \mathrm{Gr}}{2 k^{2}}\left[a k\left(f_{6}(z-1)-f_{5}(z-1)-f_{7}(z-1)+f_{8}(z-1)\right)+f_{3}(z-1)+f_{4}(z-1)\right]+ \\
+\frac{P_{1} \mathrm{Gr}}{2 k^{2}}\left[1-\left(f_{1}(z-1)+f_{2}(z-1)\right)-a k\left(f_{5}(z-1)+f_{6}(z-1)-f_{7}(z-1)-f_{8}(z-1)\right)\right],
\end{gathered}
$$

where

$$
\begin{aligned}
& f_{1}(z)=\frac{\cosh (k \delta(1+z)) \cos (k \delta(z-1))}{\cosh (2 k \delta)\left(1+2 a^{2} k^{2}\right)+2 a k(\sinh (2 k \delta)-\sin (2 k \delta))+\left(1-2 a^{2} k^{2}\right) \cos (2 k \delta)}, \\
& f_{2}(z)=\frac{\cosh (k \delta(z-1)) \cos (k \delta(z+1))}{\cosh (2 k \delta)\left(1+2 a^{2} k^{2}\right)+2 a k(\sinh (2 k \delta)-\sin (2 k \delta))+\left(1-2 a^{2} k^{2}\right) \cos (2 k \delta)}, \\
& f_{3}(z)=\frac{\sinh (k \delta(1+z)) \sin (k \delta(z-1))}{\cosh (2 k \delta)\left(1+2 a^{2} k^{2}\right)+2 a k(\sinh (2 k \delta)-\sin (2 k \delta))+\left(1-2 a^{2} k^{2}\right) \cos (2 k \delta)}, \\
& f_{4}(z)=\frac{\sin (k \delta(1+z)) \sinh (k \delta(z-1))}{\cosh (2 k \delta)\left(1+2 a^{2} k^{2}\right)+2 a k(\sinh (2 k \delta)-\sin (2 k \delta))+\left(1-2 a^{2} k^{2}\right) \cos (2 k \delta)}, \\
& f_{5}(z)=\frac{\cos (k \delta(z-1)) \sinh (k \delta(z+1))}{\cosh (2 k \delta)\left(1+2 a^{2} k^{2}\right)+2 a k(\sinh (2 k \delta)-\sin (2 k \delta))+\left(1-2 a^{2} k^{2}\right) \cos (2 k \delta)}, \\
& f_{6}(z)=\frac{\sinh (k \delta(z-1)) \cos (k \delta(z+1))}{\cosh (2 k \delta)\left(1+2 a^{2} k^{2}\right)+2 a k(\sinh (2 k \delta)-\sin (2 k \delta))+\left(1-2 a^{2} k^{2}\right) \cos (2 k \delta)}, \\
& f_{7}(z)=\frac{\cosh (k \delta(z+1)) \sin (k \delta(z-1))}{\cosh (2 k \delta)\left(1+2 a^{2} k^{2}\right)+2 a k(\sinh (2 k \delta)-\sin (2 k \delta))+\left(1-2 a^{2} k^{2}\right) \cos (2 k \delta)}, \\
& f_{8}(z)=\frac{\cosh (k \delta(z-1)) \sin (k \delta(z+1))}{\cosh (2 k \delta)\left(1+2 a^{2} k^{2}\right)+2 a k(\sinh (2 k \delta)-\sin (2 k \delta))+\left(1-2 a^{2} k^{2}\right) \cos (2 k \delta)} .
\end{aligned}
$$

Note that the dimensionless parameter $a$ is included in the expressions of velocity and pressure only with the product $a k, \quad k=\sqrt{\frac{|\sin \varphi|}{2 \mathrm{Ek}}}$. Having substituted the expression for the Ekman number Ek, we obtain $a k=\alpha \sqrt{\frac{2|\sin \varphi| \Omega}{v}}$. Thus, the product $a k$ is a dimensionless combination of the physical parameters of the system, and it is independent of the length scale $L$. 


\section{DETERMINATION OF THE EKMAN ANGLE}

Let us study the angle between the fluid velocity vector in the upper layer and the tangential stress vector. Assume that the pressure gradient is zero (isobaric flow) [1]. The tangent of the angle $\gamma$ between the velocity vector and the axis $o x$ with allowance for the Navier friction on the solid surface at a finite depth has the form

$$
\begin{aligned}
\operatorname{tg} \gamma=V(1) & / U(1)=\left[\left(\tau_{1}-\tau_{2}\right)\left(2 a k \cosh (2 k \delta)+\left(1+2 a^{2} k^{2}\right) \sinh (2 k \delta)\right)-\right. \\
& \left.-\left(\tau_{1}+\tau_{2}\right)\left(2 a k \cos (2 k \delta)+\left(1-2 a^{2} k^{2}\right) \sin (2 k \delta)\right)\right] \times \\
\times & {\left[\left(\tau_{1}+\tau_{2}\right)\left(2 a k \cosh (2 k \delta)+\left(1+2 a^{2} k^{2}\right) \sinh (2 k \delta)\right)+\right.} \\
& \left.+\left(\tau_{1}-\tau_{2}\right)\left(2 a k \cos (2 k \delta)+\left(1-2 a^{2} k^{2}\right) \sin (2 k \delta)\right)\right]^{-1}
\end{aligned}
$$

We introduce the angle $\vartheta_{a}$ such that $\vartheta_{a}=\arctan \left(\frac{2 a k \cos (2 k \delta)+\left(1-2 a^{2} k^{2}\right) \sin (2 k \delta)}{2 a k \cosh (2 k \delta)+\left(1+2 a^{2} k^{2}\right) \sinh (2 k \delta)}\right)$. After elementary transformations of the expression $\operatorname{tg} \gamma$ in Eq. (10), we have

$$
\operatorname{tg} \gamma=\frac{\frac{\tau_{1}-\tau_{2}}{\tau_{1}+\tau_{2}}-\frac{2 a k \cos (2 k \delta)+\left(1-2 a^{2} k^{2}\right) \sin (2 k \delta)}{2 a k \cosh (2 k \delta)+\left(1+2 a^{2} k^{2}\right) \sinh (2 k \delta)}}{\frac{\tau_{1}-\tau_{2}}{\tau_{1}+\tau_{2}} \times \frac{2 a k \cos (2 k \delta)+\left(1-2 a^{2} k^{2}\right) \sin (2 k \delta)}{2 a k \cosh (2 k \delta)+\left(1+2 a^{2} k^{2}\right) \sinh (2 k \delta)}+1} .
$$

It is easy to verify that the ratio $\frac{\tau_{1}-\tau_{2}}{\tau_{1}+\tau_{2}}$ is equal to $\operatorname{tg}\left(\vartheta_{\tau}-\pi / 4\right)$. Thus, by applying the tangent difference formula to expression (11), we obtain

$$
\operatorname{tg} \gamma=\operatorname{tg}\left(\vartheta_{\tau}-\vartheta_{a}-\frac{\pi}{4}\right)
$$

consequently, $\gamma=\vartheta_{\tau}-\vartheta_{a}-\pi / 4$. The angle between the velocity vector and the tangential stress vector is $\vartheta_{a}+\pi / 4$. The obtained formula is a generalization of Ekman's result [1]. Note that, when the relative depth tends to infinity, the limit of $\vartheta_{a}$ is zero. For an ocean of an infinite depth, we have

$$
\gamma=\vartheta_{\tau}-\pi / 4
$$

In this case, the angle of flow rotation is independent of the friction coefficient $a$.

Let us now calculate the angle $\vartheta_{P}$ between the pressure gradient vector $\boldsymbol{P}=\left(P_{1}, P_{2}\right)$ and the fluid velocity vector in the upper layer $\boldsymbol{V}=(U(1), V(1))$. For simplicity, we assume that the gradient vector $\boldsymbol{P}$ is directed along the latitude, i.e. $P_{1} \neq 0, P_{2}=0$. Thus, the angle between the velocity vector and the axis $o x$ is defined by the expression

$$
\vartheta_{P}=\operatorname{arctg}\left(\frac{\sinh (2 k \delta)+\sin (2 k \delta)}{\sinh (2 k \delta)-\sin (2 k \delta)+2 a k(\cosh (2 k \delta)-\cos (2 k \delta))}\right) .
$$

When the relative depth $\delta$ tends to infinity, the limit of $\vartheta_{P}$ is defined as

$$
\vartheta_{P}=\operatorname{arctg} \frac{1}{1+2 a k} \text {. }
$$


In this case, the angle of flow rotation depends on friction. At the zero Navier friction coefficient $a, \vartheta_{P}=\pi / 4$. Figure 1 shows the angle $\gamma$ as dependent on the Navier friction coefficient for different values of $k$.

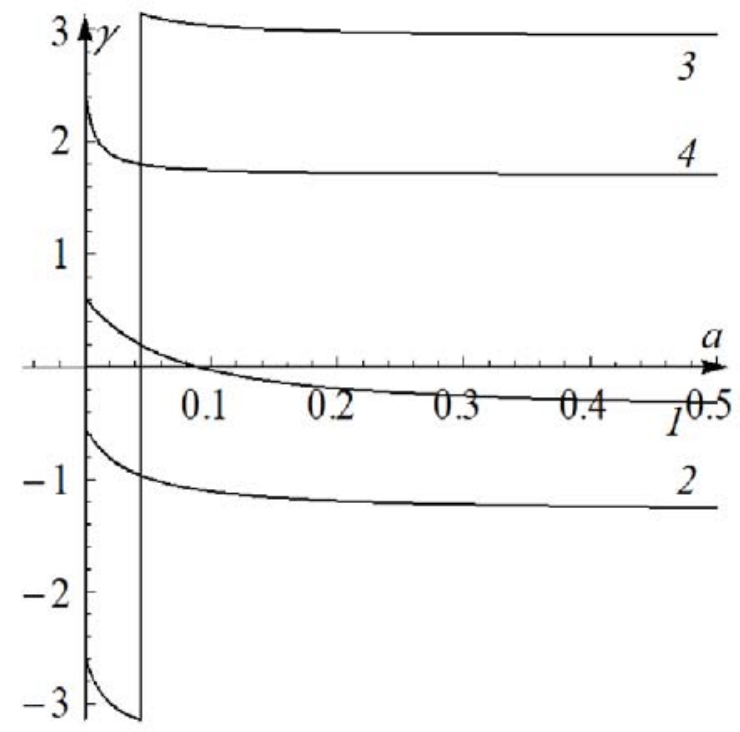

FIGURE 1. The angle $\gamma$ as dependent on the Navier friction coefficient $a$, with the numbers representing the curves corresponding to the following values of $k: k=10(1), k=20$ (2), $k=40$ (3), and $k=100$ (4)

The break in curve 3 corresponds to the transition of the value of the angle $\gamma$ from $-\pi$ to $\pi$, i.e. the velocity vector and the pressure gradient vector are contradirectional.

\section{CONCLUSION}

An analytical expression of the Ekman angle in the case of isothermal flow has been obtained. The Ekman angle has been studied analytically. It has been shown that, for a large depth, the angle is equal to $\pi / 4$. The dependence of the Ekman angle on the Navier friction coefficient $a$ and on the parameter $k$ determining the angular velocity of rotation has been constructed and studied.

\section{REFERENCES}

1. V. W. Ekman, Arkiv för Matematic, Asrtonomi, och Fysic (2)11, 1-53 (1905).

2. A. I. Felzenbaum, Theoretical Bases and Methods for Calculating Steady Sea Currents (AN USSR, Moscow, 1960).

3. J. Pedlosky, Geophysical Fluid Dynamics (Springer-Verlag, New York, 1982).

4. G. K. Korotaev, E. N. Mikhaylova, and N. B. Shapiro, Theory of Equatorial Countercurrents in the World's Ocean (Naukova Dumka, Kiev, 1986).

5. V. N. Zyryanov, Theory of Steady Ocean Currents (Gidrometeoizdat, Leningrad, 1985).

6. S. N. Aristov and E. Yu. Prosviryakov, Nonlinear Dynamics 10(2), 177-182 (2014).

7. S. N. Aristov and E. Yu. Prosviryakov, Fluid Dynamics 2, 25-31 (2016).

8. S. N. Aristov and E. Yu. Prosviryakov, Nonlinear Dynamics 9(3) 3-9 (2013).

9. S. N. Aristov and E. Yu. Prosviryakov, Theoretical Foundations of Chemical Engineering 50(3), 294-301 (2016).

10. E. Yu. Prosviryakov, Theoretical Foundations of Chemical Engineering 53 (1), 112-120 (2019).

11. S. N. Aristov and K. G. Shvarts, Vortical Flows of Advective Nature in a Rotating Fluid Layer (PSU, Perm, 2006). 
12. S. N. Aristov and K. G. Shvarts, Vortical Flows in Thin Fluid Layers (Vyatka State Univ., Kirov, 2011).

13. S. N. Aristov and K. G. Shvarts, Journal of Applied Mechanics and Technical Physics 1, 216-223 (2016).

14. L. Kh. Ingel and S. N. Aristov, Institute of Experimental Meteorology 27(162), 142-157 (1996).

15. A. V. Gorshkov and E. Yu. Prosviryakov, AIP Conference Proceedings 1915, 040020 (2017).

16. A. V. Gorshkov and E. Yu. Prosviryakov, AIP Conference Proceedings 1915, 040019 (2017).

17. A. V. Gorshkov and E. Yu. Prosviryakov, AIP Conference Proceedings 2053, 040030 (2018).

18. A. V. Gorshkov and E. Yu. Prosviryakov, AIP Conference Proceedings 2053, 040029 (2018).

19. A. V. Gorshkov and E. Yu. Prosviryakov, Izvestiya. Atmospheric and Oceanic Physics 54(2), 189-19 (2018).

20. C. Neto, D. Evans, and E. Bonaccurso, Reports on Progress in Physics 39, 2859-2897 (2005).

21. E. I. Borzenko, O. A. Dyakova, and G. P. Shrager. Vestnik TGU, Mechanics, 2(28), 35-44 (2014). 